\title{
Topological Censorship and Higher Genus Black Holes
}

\author{
G.J. Galloway, ${ }^{1}$ K. Schleich, ${ }^{2}$ D.M. Witt ${ }^{3}$ and E. Woolgar ${ }^{4}$
}

\begin{abstract}
Motivated by recent interest in black holes whose asymptotic geometry approaches that of anti-de Sitter spacetime, we give a proof of topological censorship applicable to spacetimes with such asymptotic behavior. Employing a useful rephrasing of topological censorship as a property of homotopies of arbitrary loops, we then explore the consequences of topological censorship for horizon topology of black holes. We find that the genera of horizons are controlled by the genus of the space at infinity. Our results make it clear that there is no conflict between topological censorship and the non-spherical horizon topologies of locally anti-de Sitter black holes.

More specifically, let $\mathcal{D}$ be the domain of outer communications of a boundary at infinity "scri." We show that the Principle of Topological Censorship (PTC), that every causal curve in $\mathcal{D}$ having endpoints on scri can be deformed to scri, holds under reasonable conditions for timelike scri, as it is known to do for a simply connected null scri. We then show that the PTC implies that the fundamental group of scri maps, via inclusion, onto the fundamental group of $\mathcal{D}$, i.e., every loop in $\mathcal{D}$ is homotopic to a loop in scri. We use this to determine the integral homology of preferred spacelike hypersurfaces (Cauchy surfaces or analogues thereof) in the domain of outer communications of any 4-dimensional spacetime obeying the PTC. From this, we establish that the sum of the

1 Dept. of Mathematics and Computer Science, University of Miami, Coral Gables, FL 33124, USA. e-mail:galloway@math.miami.edu

2 Dept. of Physics and Astronomy, University of British Columbia, 6224 Agriculture Road, Vancouver, BC, Canada V6T 1Z1. e-mail: schleich@noether.physics.ubc.ca

3 Dept. of Physics and Astronomy, University of British Columbia, 6224 Agriculture Road, Vancouver, BC, Canada V6T 1Z1. e-mail: donwitt@noether.physics.ubc.ca

4 Dept. of Mathematical Sciences and Theoretical Physics Institute, University of Alberta, Edmonton, AB, Canada T6G 2G1. e-mail: ewoolgar@math.ualberta.ca
\end{abstract}


genera of the cross-sections in which such a hypersurface meets black hole horizons is bounded above by the genus of the cut of infinity defined by the hypersurface. Our results generalize familiar theorems valid for asymptotically flat spacetimes requiring simple connectivity of the domain of outer communications and spherical topology for stationary and evolving black holes. 


\section{Topological Censorship and Higher Genus Black Holes}

\section{Introduction}

It is generally a matter of course that the gross features of shape, i.e., the topology, of composite objects, from molecules to stars, are determined by their internal structure. Yet black holes are an exception. A black hole has little internal structure, but the topology of its horizon is nonetheless strongly constrained, seemingly by the external structure of spacetime. This was made apparent in early work of Hawking [1], who established via a beautiful variational argument the spherical topology of stationary horizons. Hawking's proof was predicated on the global causal theoretic result that no outer trapped surfaces can exist outside the black hole region, unless energy conditions or cosmic censorship are violated. As argued by Hawking in [1], the possibility of toroidal topology, which arises as a borderline case in his argument, can be eliminated by consideration of a certain characteristic initial value problem and the assumption of analyticity; see [2] for further discussion of this issue.

In recent years an entirely different approach to the study of black hole topology has developed, based on the notion of topological censorship. In 1994, Chruściel and Wald [3], improving in the stationary setting the result on black hole topology considered in [2] and [4], were able to remove the analyticity assumption in Hawking's theorem by making use of the active topological censorship theorem of Friedman, Schleich, and Witt [5] (hereinafter, FSW). This latter result states that in a globally hyperbolic, asymptotically flat (hereinafter, AF) spacetime obeying an Averaged Null Energy Condition (ultimately, in the modified form used below — see [6]), every causal curve beginning and ending on the boundary-at-infinity could be homotopically deformed to that boundary. When topological censorship holds, the domain of outer communications (or DOC- the region exterior to black and white holes) of an AF spacetime must be simply connected [6].

Jacobson and Venkataramani [7], also using the topological censorship theorem of FSW, were able to extend the result of Chruściel and Wald on black hole topology 
beyond the stationary case. The principle behind their arguments was that any horizon topology other than spherical would allow certain causal curves outside the horizon to link with it, and so such curves would not be deformable to infinity, which would contradict FSW.

In the early 1990s, new solutions with non-spherical black hole horizons were discovered in locally anti-de Sitter (adS) spacetimes [8-13]; for a recent review, see [14]. The original topological censorship theorem did not apply to these spacetimes since they were not AF and not globally hyperbolic. However, two improvements to the proof of topological censorship indicated that these differences ought not matter. Galloway [15] was able to produce a "finite infinity" version of topological censorship that replaced the usual asymptotic conditions on the geometry with a mild geometrical condition on a finitely-distant boundary, and Galloway and Woolgar [16] were able to replace the assumption of global hyperbolicity by weak cosmic censorship. Moreover, it was soon observed [17] that topological censorship in the sense of FSW held true for each of the newly discovered black hole constructions in locally adS spacetime, although no general proof was known in this setting and obviously the aforementioned corollary implying spherical horizon topology could not hold. This is not paradoxical - the topology of the locally adS black hole spacetimes is such that no causal curve links with a non-spherical horizon in such a way as to preclude a homotopy deforming that curve to infinity. ${ }^{5}$ In fact, we will see below that the corollary implying spherical topology of black holes in AF spacetime is merely a special case of a more general corollary of topological censorship which gives a relationship between the topology of the black hole horizons and the "topology of infinity." In this sense, the topology of the black hole horizons is governed by a structure that is as "external" as possible, being entirely at infinity.

In this paper, we will consider spacetimes that obey the Principle of Topological Censorship (PTC). Let $\mathcal{M}$ be a spacetime with metric $g_{a b}$. Suppose this spacetime can

5 That topological censorship proofs should generalize to spacetimes having the asymptotic structure of these new black holes was first suggested in [7]. 
be conformally included into a spacetime-with-boundary $\mathcal{M}^{\prime}=\mathcal{M} \cup \mathcal{I}$, with metric $g_{a b}^{\prime}$ whose restriction to $\mathcal{M}$ obeys $g_{a b}^{\prime}=\Omega^{2} g_{a b}$, and where $\mathcal{I}$ is the $\Omega=0$ surface, which defines $\mathcal{I}$ as the boundary-at-infinity. For a connected boundary component $\mathcal{I},{ }^{6}$ the domain of outer communications $\mathcal{D}$ is defined by

$$
\mathcal{D}:=I^{+}(\mathcal{I}) \cap I^{-}(\mathcal{I})
$$

The PTC is the following condition on $\mathcal{D}$ :

Principle of Topological Censorship (PTC). Every causal curve whose initial and final endpoints belong to $\mathcal{I}$ is fixed endpoint homotopic to a curve on $\mathcal{I}{ }^{7}$

The PTC has already been established for general, physically reasonable AF spacetimes, cf. [5], [16]. In the next section we present a proof of the PTC in a setting that includes many asymptotically locally anti-de Sitter black hole spacetimes. This generalization exploits the fact that PTC proofs generally follow from a condition on double-null components of the Ricci tensor. These components can be related to the double-null components of the stress energy tensor through the double-null components of the Einstein equations. This relation involves no trace terms and so clearly is insensitive to the cosmological constant. This corrects the impression that the PTC is invalid in the presence of a negative cosmological constant [13,18], an impression that, if it were true, would imply that the PTC in this case would impose no constraints at all on the topology of black hole horizons. Hawking's $g=0$ restriction $[1,19,20]$ on horizon topology, by way of contrast, does assume non-negative energy density on the horizon and so is not in force in the presence of a negative cosmological constant in vacuum but, as we will see, the PTC remains valid, and imposes constraints on the topology.

${ }^{6}$ For the purpose of discussion, in the AF case we assume for simplicity that $i^{0}$ is included in $\mathcal{I}$.

7 FSW's original statement of topological censorship required every causal curve beginning and ending on $\mathcal{I}$ to be homotopic to one in a "simply connected neighborhood of infinity." Our phrasing above accomodates spacetimes for which $\mathcal{I}$ has no simply connected neighborhoods. 
In Section III, we will prove that the mapping from the fundamental group of $\mathcal{I}$ to that of the domain of outer communications is a surjection for spacetimes satisfying the PTC. This theorem generalizes previous results on the DOC being simply connected in asymptotically flat spacetimes. This characterization allows us to shift attention from causal curves to arbitrary loops in further study of the consequences of the PTC.

In Section IV, by considering loops restricted to certain spacelike surfaces and using arguments from algebraic topology, we give a very direct derivation of the topology of black hole horizons and the integral homology of hypersurfaces in exterior regions. More precisely, we consider the topology of the closure of the Cauchy surfaces or analogues thereof for the DOC whose intersections with the horizons are closed 2-manifolds ("good cuts"). We find that

$$
\sum_{i=1}^{k} g_{i} \leq g_{0}
$$

where the $g_{i}$ are the genera of the cuts of the black hole horizons and $g_{0}$ is the genus of a cut of $\mathcal{I}$ by the surface. Thus the topology of the black hole horizons is constrained by the topology at infinity. This result also pertains to any sub-domain of the DOC that lies to the future of a cut of $\mathcal{I}$ and whose Cauchy surfaces - or analogues thereofmeet the horizons at closed 2-manifolds. Therefore, it applies to the topology of the black hole horizons in the presence of black hole formation or evolution at times for which the appropriate sub-domain and surfaces can be found. Finally we demonstrate that the integral homology of these surfaces is torsion free and consequently completely determined by the Betti numbers. Furthermore, these are completely fixed in terms of the genera of the boundary.

Section $\mathrm{V}$ contains a discussion concerning these results and their applicability to the case of non-stationary black holes. 


\section{Validity of the PTC}

The aim of this section is to present a version of the PTC applicable to spacetimes which are asymptotically locally anti-de Sitter. Hence, we consider a spacetime $\mathcal{M}$, with metric $g_{a b}$, which can be conformally included into a spacetime-with-boundary $\mathcal{M}^{\prime}=\mathcal{M} \cup \mathcal{I}$, with metric $g_{a b}^{\prime}$, such that $\partial \mathcal{M}^{\prime}=\mathcal{I}$ is timelike (i.e., is a Lorentzian hypersurface in the induced metric) and $\mathcal{M}=\mathcal{M}^{\prime} \backslash \mathcal{I}$. We permit $\mathcal{I}$ to have multiple components. With regard to the conformal factor $\Omega \in C^{1}\left(\mathcal{M}^{\prime}\right)$, we make the standard assumptions that

(a) $\Omega>0$ and $g_{a b}^{\prime}=\Omega^{2} g_{a b}$ on $\mathcal{M}$, and

(b) $\Omega=0$ and $d \Omega \neq 0$ pointwise on $\mathcal{I}$.

Just as in the case of spacetimes without boundary, we say that a spacetimewith-boundary $\mathcal{M}^{\prime}$ is globally hyperbolic provided $\mathcal{M}^{\prime}$ is strongly causal and the sets $J^{+}\left(p, \mathcal{M}^{\prime}\right) \cap J^{-}\left(q, \mathcal{M}^{\prime}\right)$ are compact for all $p, q \in \mathcal{M}^{\prime}$. Note that when $\mathcal{I}$ is timelike, as in the situation considered here, $\mathcal{M}$ can never be globally hyperbolic. However in many examples of interest, such as anti-de Sitter space and the domain of outer communications of various locally adS spaces, $\mathcal{M}^{\prime}$ is.

For later convenience, we define a Cauchy surface for $\mathcal{M}^{\prime}$ to be a subset $V^{\prime} \subset \mathcal{M}^{\prime}$ which is met once and only once by each inextendible causal curve in $\mathcal{M}^{\prime}$. Then $V^{\prime}$ will be a spacelike hypersurface which, as a manifold-with-boundary, has boundary on $\mathcal{I}$. It can be shown, as in the standard case, that a spacetime-with-timelike-boundary $\mathcal{D}^{\prime}$ which is globally hyperbolic admits a Cauchy surface $V^{\prime}$ and is homeomorphic to $R \times V^{\prime}$. (This can be shown by directly modifying the proof of Prop. 6.6.8 in [19]. Alternatively, arguments can be given which involve invoking Prop. 6.6.8. For example, one can consider the "doubled spacetime" $\mathcal{M}^{\prime \prime}$ of $\mathcal{M}^{\prime}$ through $\mathcal{I}$, with metric on this double defined by the natural extension of that on $\mathcal{M}^{\prime}$. Then one can apply Prop. 6.6.8 to $\mathcal{M}^{\prime \prime}$, which will be globally hyperbolic if $\mathcal{M}^{\prime}$ is.) Many of the locally anti-de Sitter and related models $[8-11,13,14]$ which have been constructed have DOCs which admit Cauchy surfaces of this sort. 
The proof of the PTC is a consequence of the following basic result.

Theorem 2.1. Let $\mathcal{M} \subset \mathcal{M}^{\prime}$ be as described above, and assume the following conditions hold.

(i) $\mathcal{M}^{\prime}=\mathcal{M} \cup \mathcal{I}$ is globally hyperbolic.

(ii) There is a component $\mathcal{I}_{0}$ of $\mathcal{I}$ which admits a compact spacelike cut.

(iii) For each point $p$ in $\mathcal{M}$ near $\mathcal{I}_{0}$ and any future complete null geodesic $s \rightarrow \eta(s)$ in $\mathcal{M}$ starting at $p, \int_{0}^{\infty} \operatorname{Ric}\left(\eta^{\prime}, \eta^{\prime}\right) d s \geq 0$.

Then $\mathcal{I}_{0}$ cannot communicate with any other component of $\mathcal{I}$, i.e., $J^{+}\left(\mathcal{I}_{0}\right) \cap\left(\mathcal{I} \backslash \mathcal{I}_{0}\right)=\emptyset$.

Condition (iii) is a modified form of the Average Null Energy Condition (ANEC). This term usually refers to a condition of the same form as (iii) except that the integral is taken over geodesics complete to both past and future. Note that if one assumes that the Einstein equations with cosmological constant $R_{a b}-\frac{1}{2} R g_{a b}+\Lambda g_{a b}=8 \pi T_{a b}$ hold, then for any null vector $\mathrm{X}, \operatorname{Ric}(X, X)=R_{a b} X^{a} X^{b}=8 \pi T_{a b} X^{a} X^{b}$. Then the integrand $\operatorname{Ric}\left(\eta^{\prime}, \eta^{\prime}\right)$ in (iii) could be replaced by $T\left(\eta^{\prime}, \eta^{\prime}\right)$. Clearly, the presence and sign of the cosmological constant is irrelevant to whether or not a spacetime satisfying the Einstein equations will satisfy condition (iii).

For the next theorem, if $\partial \mathcal{M}^{\prime}$ is not connected, let $\mathcal{I}$ denote a single component of $\partial \mathcal{M}^{\prime}$. Let $\mathcal{D}=I^{+}(\mathcal{I}) \cap I^{-}(\mathcal{I})$ be the domain of outer communications of $\mathcal{M}$ with respect to $\mathcal{I}$. Assume that $\mathcal{D}$ does not meet any other components of $\partial \mathcal{M}^{\prime}$. Note that if the conditions of Theorem 2.1 hold, this latter assumption is automatically satisfied. Using in addition the fact that $\mathcal{I}$ is timelike, it follows that $\mathcal{D}$ is connected and the closure of $\mathcal{D}$ in $\mathcal{M}^{\prime}$ contains $\mathcal{I}$. Then $\mathcal{D}^{\prime}:=\mathcal{D} \cup \mathcal{I}$ is a connected spacetime-with-boundary, with $\partial \mathcal{D}^{\prime}=\mathcal{I}$ and $\mathcal{D}=\mathcal{D}^{\prime} \backslash \mathcal{I}$.

We now state the following topological censorship theorem, applicable to asymptotically locally adS spacetimes.

Theorem 2.2. Let $\mathcal{D}$ be the domain of outer communications with respect to $\mathcal{I}$ as described above, and assume the following conditions hold.

(i) $\mathcal{D}^{\prime}=\mathcal{D} \cup \mathcal{I}$ is globally hyperbolic. 
(ii) I admits a compact spacelike cut.

(iii) For each point $p$ in $\mathcal{M}$ near $\mathcal{I}$ and any future complete null geodesic $s \rightarrow \eta(s)$ in $\mathcal{D}$ starting at $p, \int_{0}^{\infty} \operatorname{Ric}\left(\eta^{\prime}, \eta^{\prime}\right) d s \geq 0$.

Then the PTC holds on $\mathcal{D}$.

Remark. Let $K$ be a cut of $\mathcal{I}$, and let $\mathcal{I}_{K}$ be the portion of $\mathcal{I}$ to the future of $K$, $\mathcal{I}_{K}=\mathcal{I} \cap I^{+}(K)$. Let $\mathcal{D}_{\mathcal{K}}$ be the domain of outer of communications with respect to $\mathcal{I}_{K}$, $\mathcal{D}_{\mathcal{K}}=I^{+}\left(\mathcal{I}_{K}\right) \cap I^{-}\left(\mathcal{I}_{K}\right)=I^{+}(K) \cap I^{-}(\mathcal{I})$. Theorems 2.1 and 2.2 apply equally as well to $\mathcal{D}_{\mathcal{K}}$. This procedure, first discussed by Jacobson and Venkataramani [7], allows one, by the methods of this paper, to study the topology of cuts on the future event horizon $\mathcal{H}=\partial I^{-}(\mathcal{I})$ of the form $\partial I^{+}\left(\mathcal{I}_{K}\right) \cap \mathcal{H}$. By taking $K$ sufficiently far to the future, this procedure enables one to consider cuts on $\mathcal{H}$ well to the future of the initial formation of the black hole, where one has a greater expectation that the intersection of $\mathcal{H}$ with $\partial I^{+}\left(\mathcal{I}_{K}\right)$ will be reasonable (i.e., a surface). See [7], and Sections 4 and 5 below for further discussion of this point. In what follows, it is worth keeping in mind that $\mathcal{I}$ may refer to the portion of scri to the future of a cut.

Proof of Theorem 2.1: The global hyperbolicity of $\mathcal{M}^{\prime}$ implies that $\mathcal{I}_{0}$ is strongly causal as a spacetime in its own right, and that the sets $J^{+}\left(x, \mathcal{I}_{0}\right) \cap J^{-}\left(y, \mathcal{I}_{0}\right)(\subset$ $\left.J^{+}\left(x, \mathcal{M}^{\prime}\right) \cap J^{-}\left(y, \mathcal{M}^{\prime}\right)\right)$ for $x, y \in \mathcal{I}_{0}$ have compact closure in $\mathcal{I}_{0}$. This is sufficient to imply that $\mathcal{I}_{0}$ is globally hyperbolic as a spacetime in its own right. Assumption (ii) then implies that $\mathcal{I}_{0}$ is foliated by compact Cauchy surfaces.

Now suppose that $J^{+}\left(\mathcal{I}_{0}\right)$ meets some other component $\mathcal{I}_{1}$ of $\mathcal{I}$, i.e., suppose there exists a future directed causal curve from a point $p \in \mathcal{I}_{0}$ to a point $q \in \mathcal{I}_{1}$. Let $\Sigma_{0}$ be a Cauchy surface for $\mathcal{I}_{0}$ passing through $p$. Push $\Sigma_{0}$ slightly in the normal direction to $\mathcal{I}_{0}$ to obtain a compact spacelike surface $\Sigma$ contained in $\mathcal{M}$. Let $V$ be a compact spacelike hypersurface-with-boundary spanning $\Sigma_{0}$ and $\Sigma$. By properties of the conformal factor we are assured, for suitable pushes, that $\Sigma$ is null mean convex. By this we mean that the future directed null geodesics issuing orthogonally from $\Sigma$ which are "inward pointing" with respect to $\mathcal{I}_{0}$ (i.e., which point away from $V$ ) have negative divergence. 
Under the present supposition, $J^{+}(V)$ meets $\mathcal{I}_{1}$. At the same time, $J^{+}(V)$ cannot contain all of $\mathcal{I}_{1}$. If it did, there would exist a past inextendible causal curve in $\mathcal{I}_{1}$ starting at $q \in \mathcal{I}_{1}$ contained in the compact set $J^{+}(V) \cap J^{-}(q)$, contradicting the strong causality of $\mathcal{M}^{\prime}$. Hence, $\partial J^{+}(V)$ meets $\mathcal{I}_{1}$; let $q_{0}$ be a point in $\partial J^{+}(V) \cap \mathcal{I}_{1}$. Since $\mathcal{M}^{\prime}$ is globally hyperbolic, it is causally simple; $c f$. Proposition 6.6.1 in [19], which remains valid in the present setting. Hence, $\partial J^{+}(V)=J^{+}(V) \backslash I^{+}(V)$, which implies that there exists a future directed null curve $\eta \subset \partial J^{+}(V)$ that extends from a point on $V$ to $q_{0}$. (Alternatively, one can prove the existence of this curve from results of [21], which are also valid in the present setting.) It is possible that $\eta$ meets $\mathcal{I}$ several times before reaching $q_{0}$. Consider only the portion $\eta_{0}$ of $\eta$ which extends from the initial point of $\eta$ on $V$ up to, but not including, the first point at which $\eta$ meets $\mathcal{I}$.

By properties of achronal boundaries and the conformal factor, $\eta_{0}$ is a future complete null geodesic in $\mathcal{M}$ emanating from a point on $V$. Since $\eta_{0}$ cannot enter $I^{+}(V)$, it follows that: (1) $\eta_{0}$ actually meets $V$ at a point $p_{0}$ of $\Sigma$, (2) $\eta_{0}$ meets $\Sigma$ orthogonally at $p_{0}$, and (3) $\eta_{0}$ is inward pointing with respect to $\mathcal{I}_{0}$ (i.e., $\eta_{0}$ points away from $V$ ). Since $\eta_{0}$ is future complete, the energy condition (iii) and null mean convexity of $\Sigma$ imply that there is a null focal point to $\Sigma$ along $\eta_{0}$. But beyond the focal point, $\eta_{0}$ must enter $I^{+}(\Sigma)\left(\subset I^{+}(V)\right)$, contradicting $\eta_{0} \subset \partial J^{+}(V)$.

Proof of Theorem 2.2: The proof is an application of Theorem 2.1, together with a covering space argument. Fix $p \in \mathcal{I}$. The inclusion map $i: \mathcal{I} \rightarrow \mathcal{D}^{\prime}$ induces a homomorphism of fundamental groups $i_{*}: \Pi_{1}(\mathcal{I}, p) \rightarrow \Pi_{1}\left(\mathcal{D}^{\prime}, p\right)$. The image $G=$ $i_{*}\left(\Pi_{1}(\mathcal{I}, p)\right)$ is a subgroup of $\Pi_{1}\left(\mathcal{D}^{\prime}, p\right)$. Basic covering space theory guarantees that there exists an essentially unique covering space $\widetilde{\mathcal{D}}^{\prime}$ of $\mathcal{D}^{\prime}$ such that $\pi_{*}\left(\Pi_{1}\left(\widetilde{\mathcal{D}}^{\prime}, \widetilde{p}\right)\right)=$ $G=i_{*}\left(\Pi_{1}(\mathcal{I}, p)\right)$, where $\pi: \widetilde{\mathcal{D}}^{\prime} \rightarrow \mathcal{D}^{\prime}$ is the covering map, and $\pi(\widetilde{p})=p$. Equip $\widetilde{\mathcal{D}}^{\prime}$ with the pullback metric $\pi^{*}\left(g^{\prime}\right)$ so that $\pi: \widetilde{\mathcal{D}}^{\prime} \rightarrow \mathcal{D}^{\prime}$ is a local isometry. We note that when $\mathcal{I}$ is simply connected, $\widetilde{\mathcal{D}}^{\prime}$ is the universal cover of $\mathcal{D}^{\prime}$, but in general it will not be. In a somewhat different context (i.e., when $\mathcal{D}^{\prime}$ is a spacetime without boundary and $\mathcal{I}$ is a spacelike hypersurface), $\widetilde{\mathcal{D}}^{\prime}$ is known as the Hawking covering spacetime, $c f$. [19], [22]. 
The covering spacetime $\widetilde{\mathcal{D}}^{\prime}$ has two basic properties:

(a) The component $\widetilde{\mathcal{I}}$ of $\pi^{-1}(\mathcal{I})$ which passes through $\widetilde{p}$ is a copy of $\mathcal{I}$, i.e., $\left.\pi_{*}\right|_{\widetilde{\mathcal{I}}}: \widetilde{\mathcal{I}} \rightarrow \mathcal{I}$ is an isometry.

(b) $i_{*}\left(\Pi_{1}(\widetilde{\mathcal{I}}, \widetilde{p})\right)=\Pi_{1}\left(\widetilde{\mathcal{D}}^{\prime}, \widetilde{p}\right)$, where $i: \widetilde{\mathcal{I}} \hookrightarrow \widetilde{\mathcal{D}}^{\prime}$.

Property (b) says that any loop in $\widetilde{\mathcal{D}}^{\prime}$ based at $\widetilde{p}$ can be deformed through loops based at $\widetilde{p}$ to a loop in $\widetilde{\mathcal{I}}$ based at $\widetilde{p}$. This property is an easy consequence of the defining property $\pi_{*}\left(\Pi_{1}\left(\widetilde{\mathcal{D}}^{\prime}, \widetilde{p}\right)\right)=i_{*}\left(\Pi_{1}(\mathcal{I}, p)\right)$ and the homotopy lifting property. In turn, property (b) easily implies that any curve in $\widetilde{\mathcal{D}}^{\prime}$ with endpoints on $\widetilde{\mathcal{I}}$ is fixed endpoint homotopic to a curve in $\widetilde{\mathcal{I}}$.

Now let $\gamma$ be a future directed causal curve in $\mathcal{D}^{\prime}$ with endpoints on $\mathcal{I}$. Assume $\gamma$ extends from $x \in \mathcal{I}$ to $y \in \mathcal{I}$. Let $\widetilde{\gamma}$ be the lift of $\gamma$ into $\widetilde{\mathcal{D}}^{\prime}$ starting at $\widetilde{x} \in \widetilde{\mathcal{I}}$. Note that the hypotheses of Theorem 2.1, with $\mathcal{M}=\widetilde{\mathcal{D}}:=\pi^{-1}(D), \mathcal{M}^{\prime}=\widetilde{\mathcal{D}}^{\prime}$, and $\mathcal{I}_{0}=\widetilde{\mathcal{I}}$, are satisfied. Hence the future endpoint $\widetilde{y}$ of $\widetilde{\gamma}$ must also lie on $\widetilde{\mathcal{I}}$. Then we know that $\widetilde{\gamma}$ is fixed endpoint homotopic to a curve in $\widetilde{\mathcal{I}}$. Projecting this homotopy down to $\mathcal{D}^{\prime}$, it follows that $\gamma$ is fixed endpoint homotopic to a curve in $\mathcal{I}$, as desired.

Remarks. Since many examples of locally adS spacetimes, for example those constructed by identifications of adS [9-11,13,14], do not obey the generic condition, our aim was to present a version of the PTC which does not require it. However if one replaces the energy condition (iii) by the generic condition and the ANEC: $\int_{-\infty}^{\infty} \operatorname{Ric}\left(\eta^{\prime}, \eta^{\prime}\right) d s \geq 0$ along any complete null geodesic $s \rightarrow \eta(s)$ in $\mathcal{D}$, then Theorems 2.1 and 2.2 still hold, and moreover they do not require the compactness condition (ii). The proof of Theorem 2.1 under these new assumptions involves the construction of a complete null line (globally achronal null geodesic) which is incompatible with the energy conditions. The results in this setting are rather general, and in particular the proofs do not use in any essential way that $\mathcal{I}$ is timelike. We also mention that the global hyperbolicity assumption in Theorem 2.2 can be weakened in a fashion similar to what was done in the AF case in [16].

We now turn our attention to a characterization of topological censorship which 
will allow us to more easily explore the consequences of topological censorship for black hole topology.

\section{Algebraic Characterization of Topological Censorship}

The main result of this section is a restatement of the properties of a spacetime satisfying the PTC in a language amenable to algebraic topological considerations.

Let $\mathcal{D}$ and $\mathcal{I}$ be as in Section I. Then $\mathcal{D}^{\prime}=\mathcal{D} \cup \mathcal{I}$ is a spacetime-with-boundary, with $\partial \mathcal{D}^{\prime}=\mathcal{I}$. The inclusion map $i: \mathcal{I} \rightarrow \mathcal{D}^{\prime}$ induces a homomorphism of fundamental groups $i_{*}: \Pi_{1}(\mathcal{I}) \rightarrow \Pi_{1}\left(\mathcal{D}^{\prime}\right)$. Then

Proposition 3.1. If the PTC holds for $\mathcal{D}^{\prime}$, then the group homomorphism $i_{*}: \Pi_{1}(\mathcal{I}) \rightarrow$ $\Pi_{1}\left(\mathcal{D}^{\prime}\right)$ induced by inclusion is surjective.

Remark. Note that the fundamental groups of $\mathcal{D}$ and $\mathcal{D}^{\prime}$ are trivially isomorphic, $\Pi_{1}(\mathcal{D}) \cong \Pi_{1}\left(\mathcal{D}^{\prime}\right)$. Hence, Proposition 3.1 says roughly that every loop in $\mathcal{D}$ is deformable to a loop in $\mathcal{I}$. Moreover, it implies that $\Pi_{1}(\mathcal{D})$ is isomorphic to the factor group $\Pi_{1}(\mathcal{I}) /$ ker $i_{*}$. In particular, if $\mathcal{I}$ is simply connected then so is $\mathcal{D}$.

Our proof relies on the following straightforward lemma in topology:

Lemma 3.2. Let $N$ be a manifold and $S$ an embedded submanifold with inclusion mapping $i: S \rightarrow N$. If in the universal covering space of $N$ the inverse image of $S$ by the covering map is connected, then $i_{*}: \Pi_{1}(S) \rightarrow \Pi_{1}(N)$ is surjective.

Proof: Strictly speaking, we are dealing with pointed spaces $(S, p)$ and $(N, p)$, for some fixed point $p \in S$, and we want to show that $i_{*}: \Pi_{1}(S, p) \rightarrow \Pi_{1}(N, p)$ is onto. Let $\left[c_{0}\right]$ be an element of $\Pi_{1}(N, p)$, i.e., let $c_{0}$ be a loop in $N$ based at $p$. Let $\tilde{N}$ be the universal covering space of $N$, with covering map $\pi: \tilde{N} \rightarrow N$. Choose $\tilde{p} \in \tilde{N}$ such that $\pi(\tilde{p})=p$. Let $\tilde{c}_{0}$ be the lift of $c_{0}$ starting at $\tilde{p}$; then $\tilde{c}_{0}$ is a curve in $\tilde{N}$ extending from $\tilde{p}$ to a point $\tilde{q}$ with $\pi(\tilde{q})=p$. Since $\tilde{p}, \tilde{q} \in \pi^{-1}(S)$ and $\pi^{-1}(S)$ is path connected, there exists a curve $\tilde{c}_{1}$ in $\pi^{-1}(S)$ from $\tilde{p}$ to $\tilde{q}$. The projected curve $c_{1}=\pi\left(\tilde{c}_{1}\right)$ is a loop in $S$ based at $p$, i.e., $\left[c_{1}\right] \in \Pi_{1}(S, p)$. Since $\tilde{N}$ is simply connected, $\tilde{c}_{0}$ is fixed endpoint homotopic to $\tilde{c}_{1}$. But 
this implies that $c_{0}$ is homotopic to $c_{1}$ through loops based at $p$, i.e., $i_{*}\left(\left[c_{1}\right]\right)=\left[c_{0}\right]$, as desired.

Proof of Proposition 3.1: We let $\tilde{N}$ be the universal covering spacetime of $\mathcal{N}:=\mathcal{D}^{\prime}$ with projection $\pi: \widetilde{\mathcal{N}} \rightarrow \mathcal{N}$. Then $\tilde{\mathcal{N}}=\widetilde{\mathcal{D}} \cup \widetilde{\mathcal{I}}$, where $\widetilde{\mathcal{D}}=\pi^{-1}(\mathcal{D})$ is the universal covering spacetime of $\mathcal{D}$ and $\widetilde{\mathcal{I}}=\pi^{-1}(\mathcal{I})$ is the boundary of $\tilde{N}$.

Every point in $\widetilde{\mathcal{D}}$ belongs to the inverse image by $\pi$ of some point in $\mathcal{D}$, and every point in $\mathcal{D}$ lies on some causal curve beginning and ending on $\mathcal{I}$, so every point in $\widetilde{\mathcal{D}}$ lies on some causal curve beginning and ending on $\widetilde{\mathcal{I}}$. By the PTC and basic lifting properties, no such curve can end on a different connected component of $\widetilde{\mathcal{I}}$ than it began on. Therefore, if we label these connected components by $\alpha$, the open sets $I^{+}\left(\widetilde{\mathcal{I}_{\alpha}}\right) \cap I^{-}\left(\widetilde{\mathcal{I}_{\alpha}}\right)$ are a disjoint open cover of $\widetilde{\mathcal{D}}$. But $\widetilde{\mathcal{D}}$ is connected, so $\alpha$ can take only

one value, whence $\widetilde{\mathcal{I}}$ is connected. It follows that $\mathcal{D}^{\prime}$ satisfies the conditions of Lemma 3.2 with $S=\mathcal{I}$ and therefore $i_{*}: \Pi_{1}(\mathcal{I}) \rightarrow \Pi_{1}\left(\mathcal{D}^{\prime}\right)$ is surjective. Thus $i_{*}: \Pi_{1}(\mathcal{I}) \rightarrow \Pi_{1}(\mathcal{D})$ is surjective.

All known locally anti-de Sitter black holes and related spacetimes are in accord with Proposition 3.1. We conclude this section with the following simple corollary to Proposition 3.1.

Corollary 3.3. If the PTC holds for $\mathcal{D}^{\prime}$ then $\mathcal{D}$ is orientable if $\mathcal{I}$ is.

Proof: In fact $\mathcal{D}^{\prime}$ is orientable, for if $\mathcal{D}^{\prime}$ were not orientable and $\mathcal{I}$ were, then $\mathcal{D}^{\prime}$ would possess an orientable double cover containing two copies $\mathcal{I}_{1}$ and $\mathcal{I}_{2}$ of $\mathcal{I}$. Then a curve from $p_{1} \in \mathcal{I}_{1}$ to $p_{2} \in \mathcal{I}_{2}$, where $\pi\left(p_{1}\right)=\pi\left(p_{2}\right)=p \in \mathcal{I}$, would project to a loop in $\mathcal{D}^{\prime}$ not deformable to $\mathcal{I}$, contrary to the surjectivity of $i_{*}$.

\section{Application to Black Hole Topology}

The boundary of the region of spacetime visible to observers at $\mathcal{I}$ by future directed causal curves is referred to as the event horizon. This horizon is a set of one or more null surfaces, also called black hole horizons, generated by null geodesics that have no future endpoints but possibly have past endpoints. The topology of these black 
hole horizons is constrained in spacetimes obeying the PTC because, as was seen in Section III, the topology of the domain of outer communications is constrained by the PTC - intuitively, causal curves that can communicate with observers at $\mathcal{I}$ cannot link with these horizons in a non-trivial way, rather they only carry information about the non-triviality of curves on $\mathcal{I}$.

Useful though this description is, it does not characterize the topology of these horizons themselves, but rather the hole that their excision leaves in the spacetime. However, one can obtain certain information about these horizons if one considers the topology of the intersection of certain spacelike hypersurfaces with the horizons, those whose intersection with the horizons are closed spacelike 2-manifolds (good cuts of the horizons). For example, if one has a single horizon of product form, as is the case for a stationary black hole, then the horizon topology is determined by that of the 2-manifold. Of course, black hole horizons are generally not of product form; however, the topology of any good cut is still closely related to that of the horizons. For example, if each horizon has a region with product topology, this topology is determined by that of a good cut passing through this region. As demonstrated by Jacobson and Venkataramani [7] for asymptotically flat spacetimes, the PTC constrains the topology of good cuts of the horizons by the closure of a Cauchy surface for the domain of outer communications to be 2-spheres.

Now it is possible that a black hole horizon admits two good cuts by spacelike hypersurfaces, one entirely to the future of the other, such that the cuts are not homeomorphic. This can only happen if null generators enter the horizon between the two cuts. Physically, such a situation corresponds to a black hole with transient behavior, such as that induced by formation from collapse, collision of black holes or absorption of matter. Jacobson and Venkataramani observed that their theorem could also be applied to such situations for certain spacelike hypersurfaces that cut the horizons sufficiently far away from regions of black hole formation, collision or matter absorption. Precisely, when the domain of outer communications is globally hyperbolic to the future of a cut of $\mathcal{I}$ and 
if the PTC holds on this sub-domain, then the PTC will constrain the topology of this sub-domain, of its Cauchy surface and ultimately of good cuts of the horizons. Thus, though the PTC does not determine the topology of arbitrary embedded hypersurfaces or the cuts they make on the horizons, it does do so for hypersurfaces homeomorphic to Cauchy surfaces for these sub-domains that make good cuts of the horizons. Such sub-domains can be found for black hole horizons that settle down at late times.

Below we provide a generalization of these results applicable to a more general set of spacetimes satisfying the PTC than asymptotically flat spacetimes. This generalization is based on the observation that if one can continuously push any loop in the DOC down into an appropriate spacelike surface that cuts the horizons in spacelike 2-manifolds, it follows from Prop. 3.1 that the fundamental group of the spacelike surface is related to that of $\mathcal{I}$. In the interests of a clear presentation that carefully treats all technical details, we first prove the following results for globally hyperbolic spacetimes with timelike $\mathcal{I}^{8}$ and, we emphasize, for any globally hyperbolic sub-domain corresponding to the future of a cut of $\mathcal{I}$. We then provide the results for the case of globally hyperbolic, asymptotically flat spacetimes. From the method of proof of these two cases, it is manifestly apparent that these theorems can be easily generalized to a wider class of spacetimes that satisfy the PTC. We conclude with a remark about how to provide the correct technical statement and proofs for these cases. We will also comment further on the transient behavior associated with black hole formation in the Discussion section.

Let $\mathcal{M}$ be a spacetime with timelike infinity $\mathcal{I}$ and domain of outer communications $\mathcal{D}$. Assume $\mathcal{I}$ is connected and orientable. Let $K$ be a spacelike cut of $\mathcal{I}$, and let $\mathcal{I}_{K}$ be the portion of $\mathcal{I}$ to the future of $K, \mathcal{I}_{K}=\mathcal{I} \cap I^{+}(K)$. Let $\mathcal{D}_{\mathcal{K}}$ be the DOC with respect to $\mathcal{I}_{K}, \mathcal{D}_{\mathcal{K}}=I^{+}\left(\mathcal{I}_{K}\right) \cap I^{-}\left(\mathcal{I}_{K}\right)=I^{+}(K) \cap I^{-}(\mathcal{I})$. Note $\mathcal{D}_{K}^{\prime}:=\mathcal{D}_{K} \cup \mathcal{I}_{K}$ is a connected spacetime-with-timelike-boundary, with $\partial \mathcal{D}_{\mathcal{K}}^{\prime}=\mathcal{I}_{\mathcal{K}}$ and $\mathcal{D}_{\mathcal{K}}=\mathcal{D}_{\mathcal{K}}^{\prime} \backslash \mathcal{I}_{\mathcal{K}}$. In the following we will assume that $\mathcal{D}_{\mathcal{K}}^{\prime}$ is globally hyperbolic and has a Cauchy surface

8 We remind the reader that the notions of global hyperbolicity and Cauchy surfaces for spacetimes with timelike $\mathcal{I}$ are reviewed at the beginning of Section II. 
$V^{\prime}$ as in section II. The following theorem is the main result pertaining to the topology of black holes.

Theorem 4.1. Let $\mathcal{D}_{\mathcal{K}}$ be the domain of outer communications to the future of the cut $K$ on $\mathcal{I}$ as described above. Assume $\mathcal{D}_{\mathcal{K}}^{\prime}$ is globally hyperbolic and satisfies the PTC. Suppose $V^{\prime}$ is a Cauchy surface for $\mathcal{D}_{\mathcal{K}}^{\prime}$ such that its closure $V=\overline{V^{\prime}}$ in $\mathcal{M}^{\prime}$ is a compact topological 3-manifold-with-boundary whose boundary $\partial V$ (corresponding to the edge of $V^{\prime}$ in $M^{\prime}$ ) consists of a disjoint union of compact 2-surfaces,

$$
\partial V=\bigsqcup_{i=0}^{k} \Sigma_{i},
$$

where $\Sigma_{0}$ is on $\mathcal{I}$ and the $\Sigma_{i}, i=1, \ldots, k$, are on the event horizon. Then

$$
\sum_{i=1}^{k} g_{i} \leq g_{0}
$$

where $g_{j}=$ the genus of $\Sigma_{j}, j=0,1, \ldots, k$. In particular, if $\Sigma_{0}$ is a 2 -sphere then so is each $\Sigma_{i}, i=1, \ldots, k$.

Remark 1. Many known examples of locally adS black hole spacetimes have black hole horizons with genus equal of that of scri $[9-11,13]$. In fact, for these examples, $V$ is a product space.

Remark 2. Theorem 4.1 has been stated for spacetimes with timelike $\mathcal{I}$. An analogous version for asymptotically flat spacetimes holds as well, but differs slightly in technical details. The AF case will be considered later in the section.

Theorem 4.1 is established in a series of lemmas. Lemma 4.2 connects the fundamental group of the Cauchy surface to that of $\mathcal{I}$. It is the only lemma that uses the conditions that the spacetime is globally hyperbolic and satisfies the PTC. The remaining lemmas are based purely on algebraic topology and are in fact applicable to any 3-manifold with compact boundary.

Lemma 4.2. Let the setting be as in Theorem 4.1. Then the group homomorphism $i_{*}: \Pi_{1}\left(\Sigma_{0}\right) \rightarrow \Pi_{1}(V)$ induced by inclusion $i: \Sigma_{0} \rightarrow V$ is onto. 
Proof: Let $Z^{a}$ be a timelike vector field on $\mathcal{D}_{\mathcal{K}}^{\prime}$ tangent to $\mathcal{I}_{K}$. Let $r: \mathcal{D}_{\mathcal{K}}^{\prime} \rightarrow V^{\prime}$ be the continuous projection map sending each point $p \in \mathcal{D}_{\mathcal{K}}^{\prime}$ to the unique point in $V^{\prime}$ determined by the integral curve of $Z^{a}$ through $p$. Note that $r\left(\mathcal{I}_{K}\right)=\Sigma_{0}$.

Fix $p \in \Sigma_{0}$. All loops considered are based at $p$. Let $c_{0}$ be a loop in $V$. By deforming $c_{0}$ slightly we can assume $c_{0}$ is in $V^{\prime}$. Since $\mathcal{D}_{\mathcal{K}}^{\prime}$ satisifies the hypotheses of Theorem 2.2, the PTC holds for $\mathcal{D}_{\mathcal{K}}^{\prime}$. Then, by Proposition 3.1, $c_{0}$ can be continuously deformed through loops in $\mathcal{D}_{\mathcal{K}}^{\prime}$ to a loop $c_{1}$ in $\mathcal{I}_{K}$. It follows by composition with $r$ that $c_{0}=r \circ c_{0}$ can be continuously deformed through loops in $\mathrm{V}$ to the loop $c_{2}=r \circ c_{1}$ in $\Sigma_{0}$, and hence $i_{*}\left(\left[c_{2}\right]\right)=\left[c_{0}\right]$, as desired.

By Corollary 3.3 and the assumptions of Theorem 4.1, $\mathrm{V}$ is a connected, orientable, compact 3-manifold-with-boundary whose boundary consists of $k+1$ compact surfaces $\Sigma_{i}, i=0, \ldots, k$. For the following results, which are purely topological, we assume $V$ is any such manifold.

All homology and cohomology below is taken over the integers. The $j^{\text {th }}$ homology group of a manifold $\mathcal{P}$ will be denoted by $H_{j}(\mathcal{P}), b_{j}(\mathcal{P}):=$ the rank of the free part of $H_{j}(\mathcal{P})$ will denote the $j^{\text {th }}$ Betti number of $\mathcal{P}$, and $\chi(\mathcal{P})$ will denote the Euler characteristic (the alternating sum of the Betti numbers). In the case of $b_{j}(V)\left(=b_{j}\left(V_{0}\right)\right)$, we will simply write $b_{j}$.

In general, for $V$ as assumed above, $b_{0}=1, b_{3}=0$, and $b_{1}$ and $b_{2}$ satisfy the following inequalities:

Lemma 4.3. For $V$ as above, then

(a) $b_{1} \geq \sum_{i=0}^{k} g_{i}$, and

(b) $b_{2} \geq k$.

Proof: The boundary surfaces $\Sigma_{1}, \Sigma_{2}, \ldots, \Sigma_{k}$ clearly determine $k$ linearly independent 2-cycles in $V$, and hence (b) holds. To prove (a) we use the formula, $\chi(V)=\frac{1}{2} \chi(\partial V)$, valid for any compact, orientable, odd-dimensional manifold. This formula, together with the expressions, $\chi(V)=1-b_{1}+b_{2}$ and $\chi(\partial V)=\sum_{i=0}^{k} \chi\left(\Sigma_{i}\right)=2(k+1)-2 \sum_{i=0}^{k} g_{i}$, 
implies the equation

$$
b_{1}=b_{2}+\sum_{i=0}^{k} g_{i}-k
$$

The inequality (a) now follows immediately from (b).

Lemma 4.4. If the group homomorphism $i_{*}: \Pi_{1}\left(\Sigma_{0}\right) \rightarrow \Pi_{1}(V)$ induced by inclusion is onto then the inequality (3), $\sum_{i=1}^{k} g_{i} \leq g_{0}$, holds. In particular, if $\Sigma_{0}$ is a 2-sphere then so is each $\Sigma_{i}, i=1, \ldots, k$.

Proof: We use the fact that the first integral homology group of a space is isomorphic to the fundamental group modded out by its commutator subgroup. Hence, modding out by the commutator subgroups of $\Pi_{1}\left(\Sigma_{0}\right)$ and $\Pi_{1}(V)$, respectively, induces from $i_{*}$ a surjective homomorphism from $H_{1}\left(\Sigma_{0}\right)$ to $H_{1}(V)$. It follows that the rank of the free part of $H_{1}(V)$ cannot be greater than that of $H_{1}\left(\Sigma_{0}\right)$, i.e.,

$$
b_{1} \leq b_{1}\left(\Sigma_{0}\right)=2 g_{0}
$$

Combining this inequality with the inequality (a) in Lemma 4.3 yields the inequality (3). Since $V$ is orientable, so are its boundary components. If $\Sigma_{0}$ is a 2-sphere, then each $\Sigma_{i}, i=1, \ldots, k$, is forced by $(3)$ to have genus zero, and hence is a 2 -sphere.

Proof of Theorem 4.1: Follows immediately from Lemma 4.2 and Lemma 4.4.

Next we, show that the condition on $i_{*}$ in Lemma 4.4 completely determines the homology of $V$.

Proposition 4.5. If $i_{*}: \Pi_{1}\left(\Sigma_{0}\right) \rightarrow \Pi_{1}(V)$ is onto, then the integral homology $H_{*}(V, Z)$ is torsion free, and hence is completely determined by the Betti numbers. Furthermore, the inequalities in Lemma 4.3 become equalities,

(a) $b_{1}=\sum_{i=0}^{k} g_{i}$, and

(b) $b_{2}=k$.

Proof: We first prove that $H_{*}(V, Z)$ is torsion free. Since $V$ has boundary, $H_{3}(V)=0$. Also $H_{0}(V)$ is one copy of $Z$ as $V$ is connected. Thus, we need to show $H_{1}(V)$ and $H_{2}(V)$ are free. 
Claim 1. $H_{2}(V)$ is free.

To prove Claim 1 we recall the classic result that $H_{n-1}\left(N^{n}\right)$ is free for an orientable closed n-manifold $N^{n}$. To make use of this, let $V^{\prime}$ be a compact orientable 3-manifold without boundary containing $V$ (e.g., take $V^{\prime}$ to be the double of $V$ ), and let $B=V^{\prime} \backslash V$.

Assume that $W$ is a non-trivial torsion element in $H_{2}(V)$. Now view $W$ as an element in $H_{2}\left(V^{\prime}\right)$. Suppose $W=0$ in $H_{2}\left(V^{\prime}\right)$. Then $W=0$ in $H_{2}\left(V^{\prime}, B\right)$. By excision, $H_{2}\left(V^{\prime}, B\right)=H_{2}(V, \partial V)$, where $\partial V$ is the manifold boundary of $V$. Hence $W=0$ in $H_{2}(V, \partial V)$. This means that $W=$ a sum of boundary components in $H_{2}(V)$. But a sum of boundary components cannot be a torsion element. Thus, $W \neq 0$ in $H_{2}\left(V^{\prime}\right)$. Moreover, if $n W=0$ in $H_{2}(V)$ then $n W=0$ in $H_{2}\left(V^{\prime}\right)$. It follows that $W$ is a non-trivial torsion element in $H_{2}\left(V^{\prime}\right)$, a contradiction. Hence, $H_{2}(V)$ is free.

Claim 2. $H_{1}(V)$ is free.

To prove Claim 2 we first consider the relative homology sequence for the pair $V \supset \Sigma_{0}$,

$$
\cdots \quad \rightarrow H_{1}\left(\Sigma_{0}\right) \stackrel{\alpha}{\rightarrow} H_{1}(V) \stackrel{\beta}{\rightarrow} H_{1}\left(V, \Sigma_{0}\right) \stackrel{\partial}{\rightarrow} \tilde{H}_{0}\left(\Sigma_{0}\right)=0 \quad .
$$

(Here $\tilde{H}_{0}\left(\Sigma_{0}\right)$ is the reduced zeroeth-dimensional homology group.) Since, as discussed in Lemma 4.4, $\alpha$ is onto, we have $\operatorname{ker} \beta=\operatorname{im} \alpha=H_{1}(V)$ which implies $\beta \equiv 0$. Hence $\operatorname{ker} \partial=\operatorname{im} \beta=0$, and thus $\partial$ is injective. This implies that $H_{1}\left(V, \Sigma_{0}\right)=0$.

Now consider the relative homology sequence for the triple $V \supset \partial V \supset \Sigma_{0}$,

$$
\cdots \quad \rightarrow H_{1}\left(\partial V, \Sigma_{0}\right) \rightarrow H_{1}\left(V, \Sigma_{0}\right)=0 \rightarrow H_{1}(V, \partial V) \stackrel{\partial}{\rightarrow} H_{0}\left(\partial V, \Sigma_{0}\right) \rightarrow \cdots .
$$

Since $H_{0}\left(\partial V, \Sigma_{0}\right)$ is torsion free and $\partial$ is injective, $H_{1}(V, \partial V)$ is torsion free. Next, Poincaré-Lefschetz duality gives $H^{2}(V) \cong H_{1}(V, \partial V)$. Hence $H^{2}(V)$ is torsion free. The universal coefficient theorem implies that

$$
H^{2}(V) \cong \operatorname{Hom}\left(H_{2}(V), Z\right) \oplus \operatorname{Ext}\left(H_{1}(V), Z\right)
$$

The functor $\operatorname{Ext}(-,-)$ is bilinear in the first argument with respect to direct sums and $\operatorname{Ext}\left(\mathrm{Z}_{\mathrm{k}}, \mathrm{Z}\right)=Z_{k}$. Hence $H^{2}(V)$ cannot be torsion free unless $H_{1}(V)$ is. This completes the proof of Claim 2 and the proof that $H_{*}(V)$ is torsion free. 
It remains to show that the inequalities in Lemma 4.3 become equalities. We prove $b_{2}=k$; the equation $b_{1}=\sum_{i=0}^{k} g_{i}$ then follows from equation (4). In view of Lemma 4.3, it is sufficient to show that $b_{2} \leq k$. Since $H_{2}(V)$ is finitely generated and torsion free, we have $H_{2}(V) \cong H^{2}(V) \cong H_{1}(V, \partial V)$, where we have again made use of Poincaré-Lefschetz duality. Hence, $b_{2}=\operatorname{rank} H_{1}(V, \partial V)$. To show that rank $H_{1}(V, \partial V) \leq k$, we refer again to the long exact sequence (7). By excision, $H_{0}\left(\partial V, \Sigma_{0}\right) \cong H_{0}\left(\partial V \backslash \Sigma_{0}, \emptyset\right)=H_{0}\left(\partial V \backslash \Sigma_{0}\right)$. Hence, by the injectivity of $\partial, \operatorname{rank} H_{1}(V, \partial V) \leq \operatorname{rank} H_{0}\left(\partial V, \Sigma_{0}\right)=$ the number of components of $\partial V \backslash \Sigma_{0}=k$. This completes the proof of Proposition 4.5.

The conclusion of Proposition 4.5 applies to the spacelike 3 -surface-with-boundary $V$ of Theorem 4.1. Thus, we have completely determined the homology of the Cauchy surfaces of $\mathcal{D}_{\mathcal{K}}^{\prime}$.

We now consider the asymptotically flat case with null infinity $\mathcal{I}=\mathcal{I}^{+} \cup \mathcal{I}^{-}$. For this case, let $K$ be a spacelike cut of $\mathcal{I}^{-}$, and let $\mathcal{I}_{K}$ be the portion of $\mathcal{I}$ to the future of $K, \mathcal{I}_{K}=\mathcal{I} \cap J^{+}(K)$. Let $\mathcal{D}_{\mathcal{K}}$ be the domain of outer of communications with respect to $\mathcal{I}_{K}, \mathcal{D}_{\mathcal{K}}=I^{+}\left(\mathcal{I}_{K}\right) \cap I^{-}\left(\mathcal{I}_{K}\right)=I^{+}(K) \cap I^{-}\left(\mathcal{I}^{+}\right) . \mathcal{D}_{K}^{\prime}:=\mathcal{D}_{K} \cup \mathcal{I}^{+}$is a connected spacetime-with-boundary, with $\partial \mathcal{D}_{\mathcal{K}}^{\prime}=\mathcal{I}^{+}$and $\mathcal{D}_{\mathcal{K}}=\mathcal{D}_{\mathcal{K}}^{\prime} \backslash \mathcal{I}^{+}$.

We then have the following analogue of Theorem 4.1 .

Theorem 4.1'. Let $\mathcal{D}_{\mathcal{K}}$ be the domain of outer communications to the future of the cut $K$ on $\mathcal{I}^{-}$of an asymptotically flat spacetime $\mathcal{M}$ as described above. Assume $\mathcal{D}_{\mathcal{K}}^{\prime}$ is globally hyperbolic and satisfies the PTC. Suppose $V_{0}$ is a Cauchy surface for $\mathcal{D}_{\mathcal{K}}$ such that its closure $V=\overline{V_{0}}$ in $\mathcal{M}$ is a topological 3-manifold-with-boundary, compact outside a small neighborhood of $i^{0}$, with boundary components consisting of a disjoint union of compact 2-surfaces,

$$
\partial V=\bigsqcup_{i=1}^{k} \Sigma_{i}
$$

where the $\Sigma_{i}, i=1, \ldots, k$, are on the event horizon. Then all $\Sigma_{i}, i=1, \ldots, k$ are 2-spheres. Moreover, $V_{0}$ has the topology of a homotopy 3 -sphere minus $k+1$ closed 3-balls. 
Remark 1. In the AF case the asymptotic topology is spherical, which corresponds to $g_{0}=0$ in inequality (3). But since $g_{i}=0, i=1, \ldots, k$, inequality (3) is satisfied in the AF case, as well. Again, the topology of the event horizon is constrained by the topology at infinity.

Remark 2. This theorem is a slightly strengthened version of the main theorem in [7]; it does not assume orientability of $V_{0}$ and we conclude a stronger topology for this Cauchy surface.

Proof of Theorem 4.1': The arguments used to prove Theorem 4.1 can be easily adapted, with only minor technical changes involved, to prove in the AF case that each $\Sigma_{i}$ is a 2 -sphere. Alternatively, one may argue as follows. By known results on topological censorship in the AF case $([5],[6]), \mathcal{D}_{\mathcal{K}}$ is simply connected, and hence so is $V_{0}$. It follows that $\tilde{V}=V \cup\left\{i^{0}\right\}$ is a compact simply connected 3-manifold-withboundary, with boundary components $\Sigma_{i}, i=1, \ldots, k$. Then, according to Lemma 4.9 , p. 47 in Hempel [23], each $\Sigma_{i}$ is a 2 -sphere. By attaching 3-cells to each $\Sigma_{i}$ we obtain a closed simply connected 3 -manifold, which by well-known results (see [23]) is a homotopy 3 -sphere. Removing the attached 3-cells and $i_{0}$ we obtain that $V_{0}$ is a homotopy 3 -sphere minus $k+1$ closed balls.

Remark. Although the above results were proved assuming global hyperbolicity, it is clear that the same results will hold for a more general set of spacetimes that satisfy the PTC and for which a version of Lemma 4.2 can be proved. Spacetimes that are not globally hyperbolic but satisfy a weaker condition such as weak cosmic censorship can still admit a projection onto a preferred spacelike surface. In particular, one can generalize the projection given by the integral flow of a timelike vector field on the domain of outer communications used to push loops into the Cauchy surface to be a retract. Recall a retract of $X$ onto a subspace $A$ is a continuous map $r: X \rightarrow A$ such that $\left.r\right|_{A}=$ id. Thus, if $V_{0}$ is a regular retract of $\mathcal{D}$, that is, if there exists a retract $r: \mathcal{D} \cup \mathcal{I} \rightarrow V_{0} \cup \Sigma_{0}$ such that $r(\mathcal{I}) \subseteq \Sigma_{0}$, then one can again establish Lemma 4.2. 


\section{Discussion}

We wish to emphasize that the results concerning black hole topology obtained in Section IV in no way contradict the numerical findings of [24] concerning the existence in principle of temporarily toroidal black holes in asymptotically flat spacetimes. The consistency of topological censorship with asymptotically flat models containing temporarily toroidal black hole horizons has been clearly elucidated in [25]. The acausal nature of cross-over sets, expected to be present in the early formation of the event horizon, permits slicings of the event horizon in asymptotically flat black hole spacetimes with exotic (i.e., non-spherical) topologies. See the recent papers $[26,27]$ for further discussion. As described in Section IV, the method of topological censorship for exploring the topology of black hole horizons makes use of specific time slices, namely, Cauchy surfaces for the DOC or for the sub-region of the DOC to the future of a cut on $\mathcal{I}$. Surfaces exhibiting temporarily toroidal black hole horizons are not such surfaces. Moreover, the method requires such a slice to have non-empty edge which meets the horizons in $C^{0}$ compact surfaces. We elaborate further on these points below.

It is important to keep in mind that not all Cauchy surfaces $V_{0}$ for the DOC are interiors of orientable manifolds with boundary $V$ corresponding to the intersection of a spacelike slice with the black hole horizons. Consider the $t=0$ slice of the $R P^{3}$ geon. As discussed in [5], this spacetime is constructed from the $t=0$ slice of Schwarzschild spacetime by identifying antipodal points at the throat $r=2 M$. The maximal evolution of this slice is a spacetime with spatial topology $R P^{3}-p t$. Its universal covering space is the maximally extended Schwarzschild spacetime.

The $t=0$ slice of the $R P^{3}$ geon contains a non-orientable $R P^{2}$ with zero expansion. This $R P^{2}$ is not a trapped surface as it does not separate the slice into two regions. It is not part of the DOC as any radially outward directed null geodesic from this surface does not intersect $\mathcal{I}$; thus it is clearly part of the horizon. The intersection of the DOC with the $t=0$ slice produces a simply connected $V_{0}$. The intersection of the horizon with this slice is $R P^{2}$. However, we cannot attach this surface to $V_{0}$ to produce a manifold 
with boundary $V$ by the inclusion map; instead, this map reproduces the original $t=0$ slice which has no interior boundary. Note, however, that any spacelike slice that does not pass through this $R P^{2}$ will intersect the horizon at an $S^{2}$. In fact, this will be the generic situation. Moreover, the intersection of such a slice with the DOC will produce a simply connected $V_{0}$ which is the interior of a closed connected orientable $V$ with an $S^{2}$ interior boundary.

Clearly this example does not contradict any results of Section IV, which assumes an orientable $V$ with two or more boundaries. However, it does yield the important lesson that one must construct $V$ to apply the theorem, not $V_{0}$. It also gives an example of a badly behaved cut of the horizon, again illustrating the usefulness of taking slices to the future of a cut of $\mathcal{I}$.

For our second example, we construct a toy model of a black hole spacetime that mimics a special case of topology change, namely that of black hole formation from a single collapse. This model illustrates several features; how a choice of a hypersurface can affect the description of horizon topology and how some cuts of $\mathcal{I}$ give rise to a Cauchy surface whose edge on the horizon is not a 2-manifold.

We begin with a 3-dimensional model; later a 4-dimensional example will be constructed by treating the 3-dimensional model as a hyperplane through an axis of symmetry in the larger spacetime. Our spacetime can have either anti-de Sitter or flat geometry; as both are conformal to regions of the Einstein static cylinder, we use as coordinates in the construction below those of the conformally related flat metric ( $c f$. [19], sections 5.1 and 5.2). We depict $\mathcal{I}$ as timelike in the accompanying figures, but it can equally well be null.

We begin in 3 dimensions with a line segment $L$ defined by $t=y=0,|x| \leq l$. The future $I^{+}(L)$ of this line segment is a sort of elongated cone, whose traces in hyperplanes of constant $t$ have the shape of rectangles with semi-circular caps attached to the two short sides. We foliate the spacetime by hyperboloids,

$$
t=a+\sqrt{r^{2}+b}
$$


where $r^{2}=x^{2}+y^{2}$ and $a, b$ are conveniently chosen parameters. These hyperboloids cut $\mathcal{I}$ in a circle. We now remove from spacetime all points of $J^{+}(L)$ above a hyperplane $t=d$ intersecting it to the future. What remains is a black hole spacetime and has a globally hyperbolic domain of outer communications $\mathcal{D}$ (again, $c f$. Section II). The black hole is the set of remaining points of $I^{+}(L)$. The horizon $\partial I^{-}\left(\mathcal{I}^{+}\right)$is generated by null geodesics that all begin on $L$.

The Cauchy surface for $\mathcal{D}$ will have topology $R^{2}$ and does not cross the horizon. Thus, to probe the topology of the horizon, one needs to consider spacetimes corresponding to the future of a cut of $\mathcal{I}$. However, not every cut of $\mathcal{I}$ will produce a spacetime with a Cauchy slicing with the correct properties. Such a bad cut of $\mathcal{I}$ is illustrated in Figure 1. The boundary of the causal future of this cut intersects the horizon at a segment $I$ of $L$. The topology of a Cauchy slice for its DOC is $R^{2} \backslash I$. Its closure intersects the horizon at $I$; thus, as in the $R P^{3}$ geon above, the closure of this slice has no inner boundary, being in this case $R^{2}$.

This 3-d spacetime corresponds to a 4- $\mathrm{d}$ axisymmetric spacetime. The correspondence between axisymmetric spatial hypersurfaces and the $x y$ planes of the spacetime is generated by rotating each $x y$ plane about the $y$ axis. After this rotation, one sees that the line segment $L$ becomes a disk in the 4-d axisymmetric spacetime. The Cauchy surface in question meets the horizon in a disk (a closed 2-ball) and has topology $R^{3}$ minus that disk. The closure of the Cauchy surface is $R^{3}$ and again has no internal boundary. Thus the results of Section IV do not apply to this Cauchy slice.

A good cut of $\mathcal{I}$ is illustrated in Figure 2. This cut intersects the horizon at a sphere. The topology of a Cauchy surface for its DOC is $R^{2} \backslash \overline{B^{2}}$ and the intersection of its closure is $S^{1}$. The Cauchy surface in the corresponding 4-dimensional model has topology $R^{3} \backslash \overline{B^{3}}$ with internal boundary $S^{2}$. The results of Section IV clearly apply in the latter case.

Of course, not all spacelike surfaces need be Cauchy surfaces of the spacetime to the future of a cut of $\mathcal{I}$. The family of hyperboloids (9), two of which are illustrated 
in Figure 3, provides an example of such surfaces. As recognized in [25] in a similar model, this family exhibits formation of a temporarily toroidal black hole horizon as the parameter $a$ in (9) increases; these surfaces intersect the horizon in a pair of topological circles, which by axial symmetry correspond to a toroidal horizon in the 4-dimensional spacetime. The circles increase in size and eventually meet, whence the horizon topology changes. After this point, these surfaces meet the horizon at a circle, corresponding to a sphere by axial symmetry.

In contrast, with respect to constant- $t$ surfaces, the horizon forms completely at the $t=0$ instant. For every $t>0$ hypersurface, the black hole has spherical topology, and inequality (3) holds. Any $t=t_{0}>0$ hypersurface is a Cauchy surface for the region of $\mathcal{D}$ that lies in the future of an appropriate cut of $\mathcal{I}$. The apparent change of horizon topology from toroidal to spherical was an effect entirely dependent on the choice of hypersurface. The only unambiguous description of this black hole is that no causal curve was able to link with the horizon; i.e., that the PTC was not violated.

In the introduction, we offered the view that the topology of the boundary at infinity constrained that of the horizons, but one could equally well reverse this picture. Let us contemplate a black hole considered as a stationary, causally well-behaved, isolated system cut off from the Universe by a sufficiently distant boundary- $\mathcal{I}$. Then we have shown that topological censorship requires the genus of the horizon to be a lower bound for that of the boundary. As remarked above, this seems an intuitive result; as illustrated in Figure 4, when one visualizes placing a genus $g_{1}$ surface within a genus $g_{2}$ "box" with no possibility of entangling curves, it seems clear that $g_{2} \geq g_{1}$. Yet, as is often the case with such things, the powerful machinery of algebraic topology was required to prove it. An advantage of using this powerful tool is that we were able to completely specify the homology of well-behaved exterior regions of black holes and, in virtue of Proposition 4.5, to say that all interesting homology of these exteriors, save that which is reflected in the topology of scri, is directly attributable to the presence of horizons. 


\section{Acknowledgements}

EW wishes to acknowledge conversations with R.B. Mann and W. Smith concerning locally anti-de Sitter spacetimes. GJG and EW thank Piotr Chruściel for conversations concerning the validity of topological censorship for these spacetimes. This work

was partially supported by the Natural Sciences and Engineering Research Council of Canada and by the National Science Foundation (USA), Grant No. DMS-9803566.

\section{References}

[1] S.W. Hawking, Commun. Math. Phys. 25 (1972), 152.

[2] G.J. Galloway, in Differential Geometry and Mathematical Physics, Contemporary Math. 170, ed. J. Beem and K. Duggal (AMS, Providence, 1994), 113.

[3] P.T. Chruściel and R.M. Wald, Class. Quantum Gravit. 11 (1994), L147.

[4] S.F. Browdy and G.J. Galloway, J. Math. Phys. 36 (1995), 4952.

[5] J.L. Friedman, K. Schleich, and D.M. Witt, Phys. Rev. Lett. 71 (1993), 1486.

[6] G.J. Galloway, Class. Quantum Gravit. 12 (1995), L99.

[7] T. Jacobson and S. Venkataramani, Class. Quantum Gravit. 12 (1995), 1055.

[8] J.P.S. Lemos, Phys. Lett. B 352 (1995), 46.

[9] M. Bañados, C. Teitelboim, and J. Zanelli, Phys. Rev. Lett. 69 (1992), 1849;

M. Bañados, M. Henneaux, C. Teitelboim, and J. Zanelli, Phys. Rev. D48 (1993), 1506.

[10] S. Åminneborg, I. Bengtsson, S. Holst, and P. Peldán, Class. Quantum Gravit. 13 (1996), 2707.

[11] R.B. Mann, Class. Quantum Gravit. 14 (1997), 2927

12 W.L. Smith and R.B. Mann, Phys. Rev. D 56 (1997), 4942.

[13] D.R. Brill, J. Louko, and P. Peldán, Phys. Rev. D 56 (1997), 3600.

[14] R.B. Mann, in Internal Structure of Black Holes and Spacetime Singularities, eds. L. Burko and A. Ori, Ann. Israeli Phys. Soc. 13 (1998), 311.

[15] G.J. Galloway, Class. Quantum Gravit. 13 (1996), 1471. 
[16] G.J. Galloway and E. Woolgar, Class. Quantum Gravit. 14 (1997), L1.

[17] S. Åminneborg, I. Bengtsson, D. Brill, S. Holst, and P. Peldán, Class. Quantum Gravit. 15 (1998), 627.

[18] L. Vanzo, Phys. Rev. D 56 (1997), 6475.

[19] S.W. Hawking and G.F.R. Ellis, The Large Scale Structure of Space-Time, (Cambridge University Press, Cambridge, 1973).

[20] E. Woolgar, in progress.

[21] R.D. Sorkin and E. Woolgar, Class. Quantum Gravit. 13 (1996), 1971.

[22] B.C. Haggman, G.W. Horndeski, and G. Mess, J. Math. Phys. 21 (1980), 2412.

[23] J. Hempel, 3-Manifolds, (Princeton University Press, Princeton, 1976).

[24] S.A. Hughes et al., Phys. Rev. D 49 (1994), 4004.

[25] S.L. Shapiro, S.A. Teukolsky, and J. Winicour, Phys. Rev. D 52 (1995), 6982.

[26] S. Husa and J. Winicour, preprint gr-qc/9905039.

[27] M. Siino, Phys. Rev. D 58 (1998), 104016; D 59 (1999), 064006. 
Figures

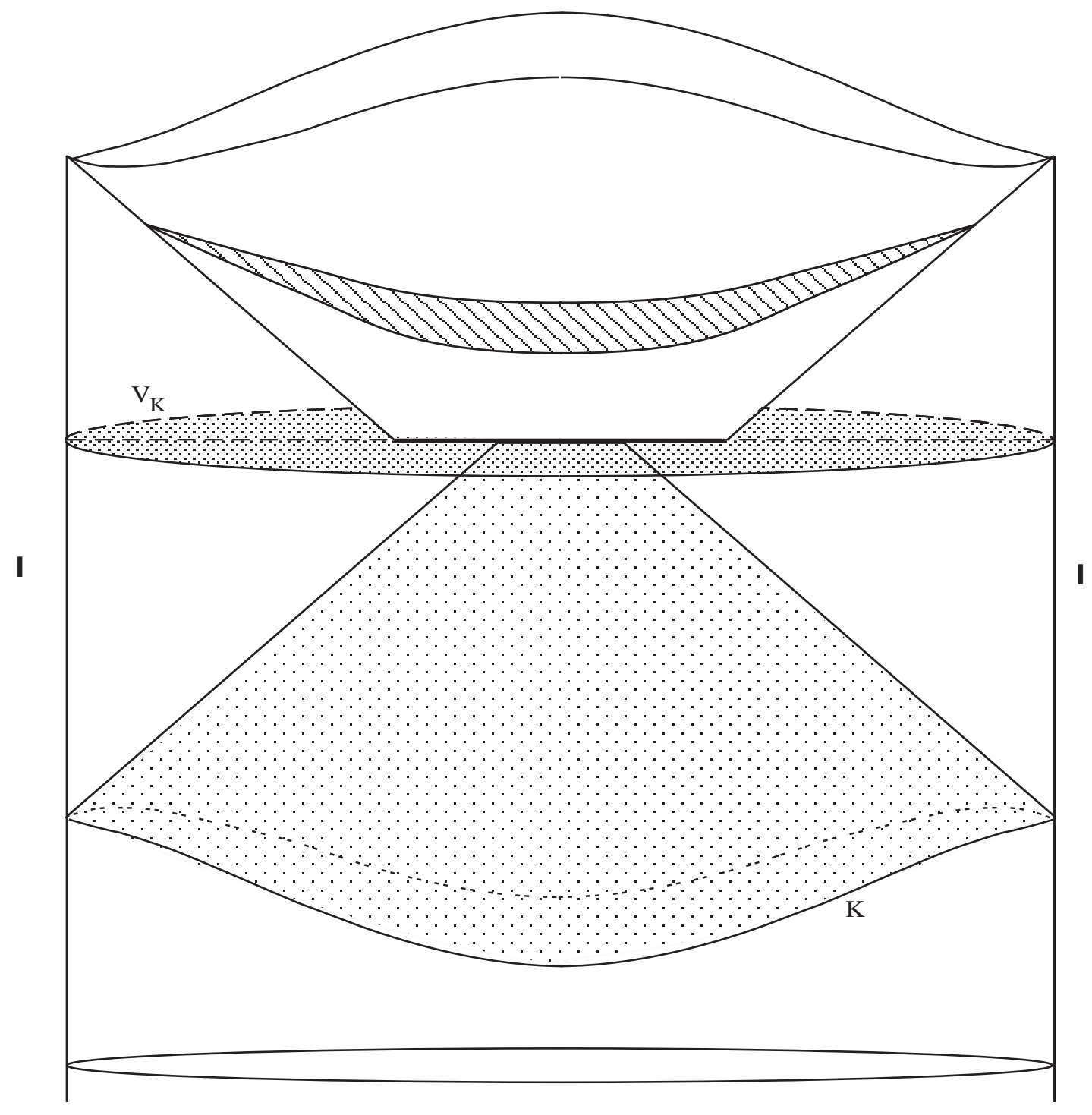

Figure 1. A bad cut of the horizon. The surface $V_{K}$ is the Cauchy surface for the domain of outer communications of the spacetime to the future of the cut $K$ of $\mathcal{I}$. The inner boundary of the causal future of $K$ intersects the horizon at a spacelike line segment. Consequently the closure of $V_{K}$ has topology $R^{2}$. 


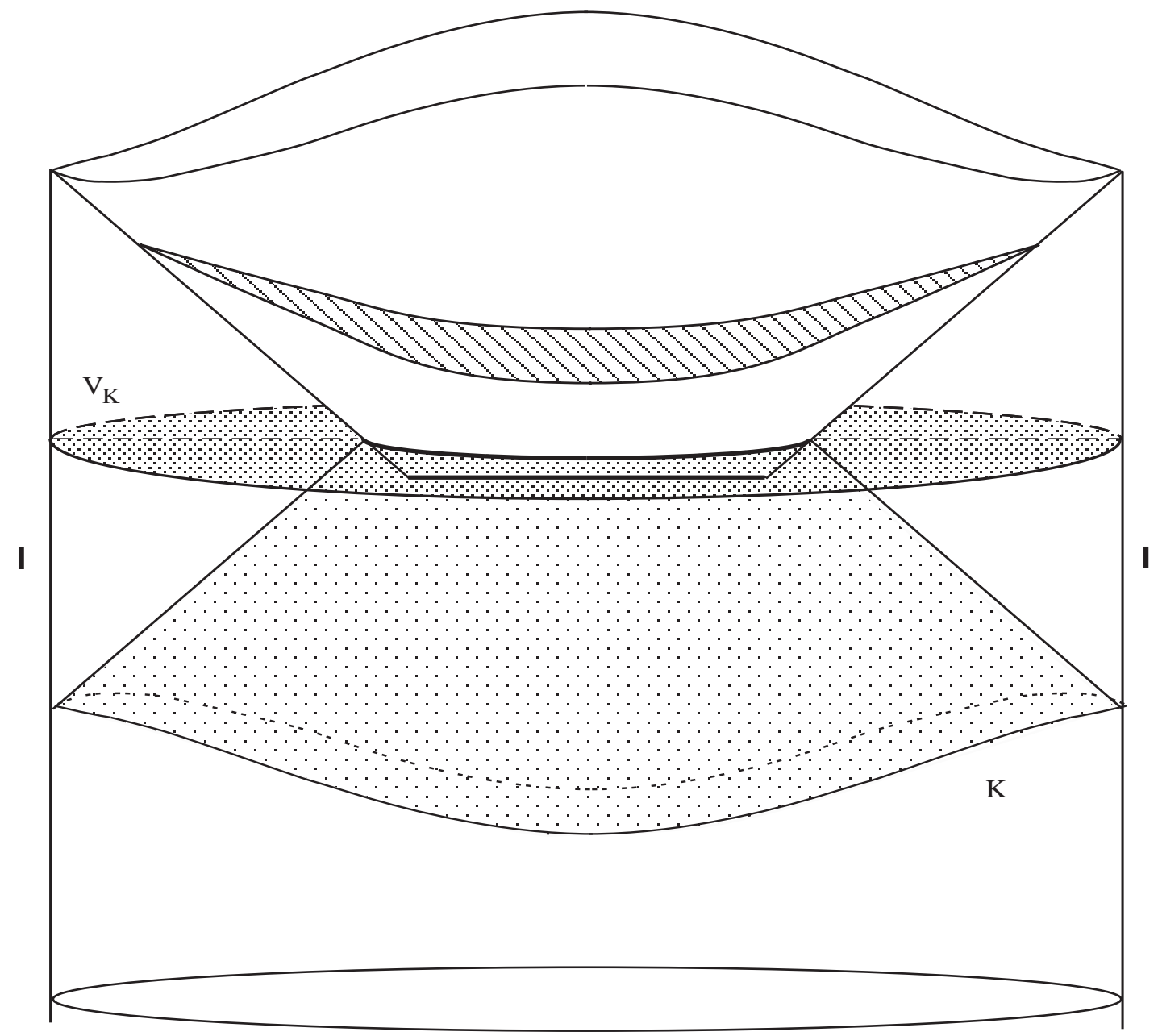

Figure 2. A good cut of the horizon. The surface $V_{K}$ is the Cauchy surface for the domain of outer communications of the spacetime to the future of the cut $K$ of $\mathcal{I}$. The intersection of the inner boundary of the causal future of $K$ with the horizon is now $S^{1}$. Consequently the closure of $V_{K}$ intersects the horizon at $S^{1}$. 

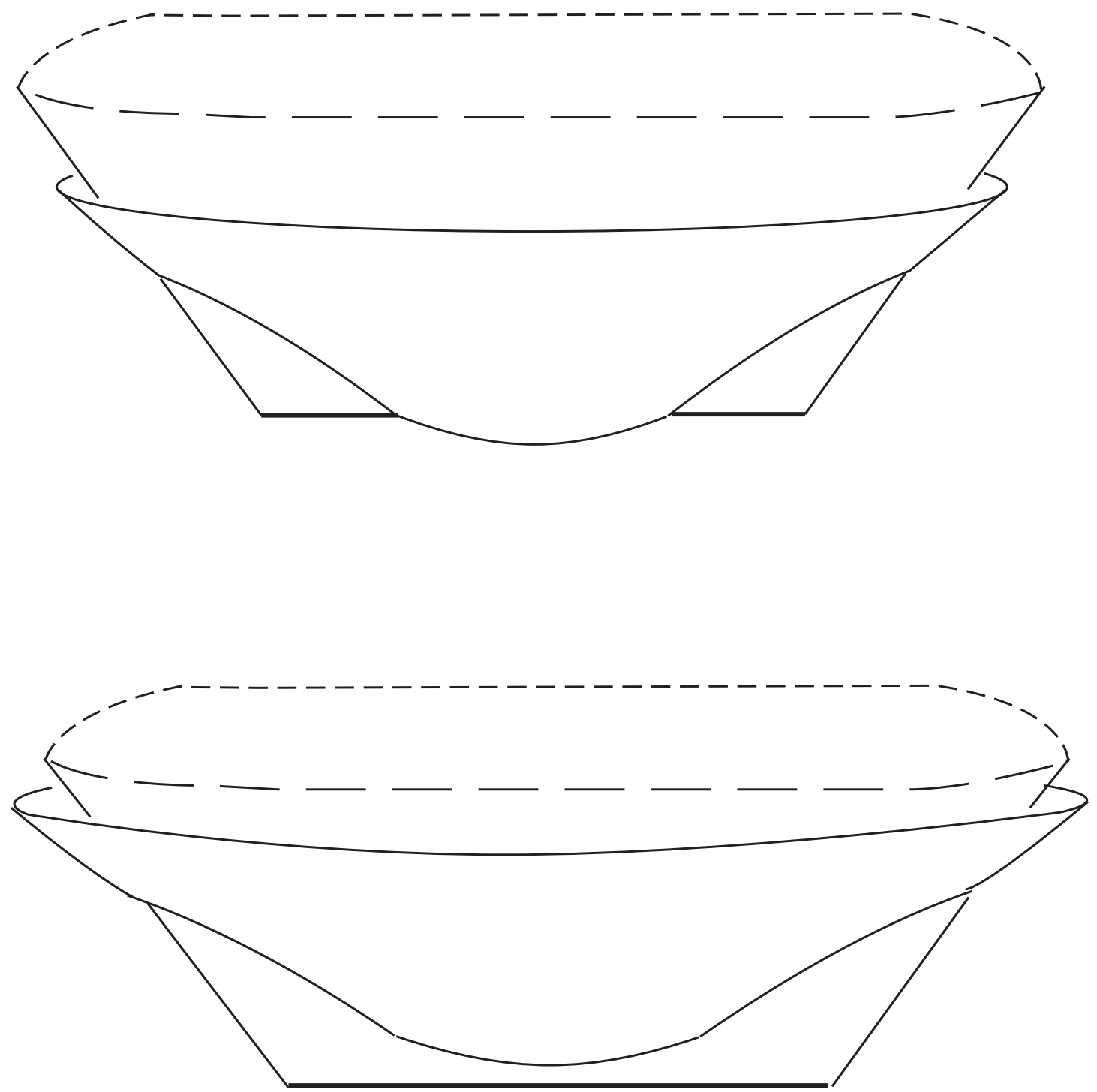

Figure 3. Two slicings of the horizon by hyperboloids. Both illustrations concentrate on the region near the horizon. The top illustration is of a hyperboloid that intersects the horizon at two topological circles. The bottom illustration is of a hyperboloid that lies to the future of the first. It intersects the horizon at one topological circle. 

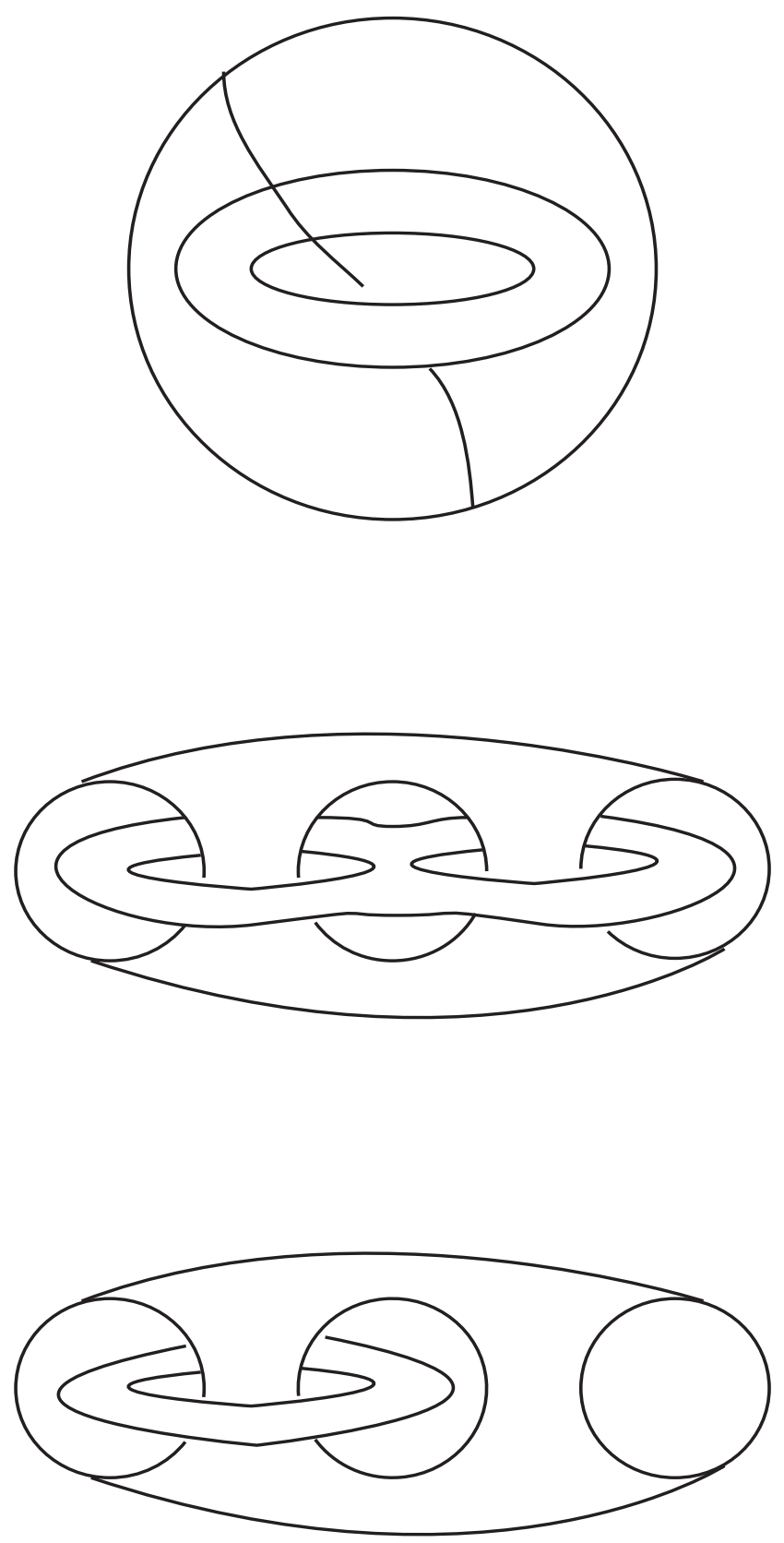

Figure 4. Entanglement and non-entanglement of curves on black hole horizons. Each illustration displays a cross-section of a cut of $\mathcal{I}$. The cut-away reveals a cut of a black hole horizon inside. In the top illustration, the cut of $\mathcal{I}$ has genus zero, that of the horizon has genus 1, and, as illustrated, there are curves not deformable to $\mathcal{I}$. In the middle illustration, the genus of the cut of $\mathcal{I}$ and that of the horizon are both 2 , and they are linked in such a manner that every curve is deformable to $\mathcal{I}$. In the illustration at bottom, the genus of the cut of $\mathcal{I}$ exceeds that of the horizon, and again every curve is deformable to $\mathcal{I}$. 